\title{
Some new iterative algorithms with errors for common solutions of two finite families of accretive mappings in a Banach space
}

\section{Li Wei ${ }^{*}$ and Ruilin Tan}

\section{"Correspondence:}

diandianba@yahoo.com

School of Mathematics and

Statistics, Hebei University of

Economics and Business,

Shijiazhuang, 050061, China

\begin{abstract}
The purpose of this paper is to prove some new theorems of strong convergence to common solutions for two finite families of accretive mappings in a real uniformly smooth and uniformly convex Banach space by means of some new iterative algorithms with errors, which extend the corresponding works by some authors. As applications, the theorems of strong convergence to common fixed points of two finite families of pseudo-contractive mappings are presented.
\end{abstract}

MSC: $47 \mathrm{H} 05 ; 47 \mathrm{H} 09 ; 47 \mathrm{H} 10$

Keywords: accretive mapping; pseudo-contractive mapping; common zeros; uniformly smooth (or convex) Banach space

\section{Introduction}

Let $E$ be a real Banach space with norm $\|\cdot\|$ and let $E^{*}$ denote the dual space of $E$. We use $\rightarrow$ and $\rightarrow$ to denote strong and weak convergence, respectively. We denote the value of $f \in E^{*}$ at $x \in E$ by $\langle x, f\rangle$.

We use $J$ to denote the normalized duality mapping from $E$ to $2^{E^{*}}$, which is defined by

$$
J x:=\left\{f \in E^{*}:\langle x, f\rangle=\|x\|^{2}=\|f\|^{2}\right\}, \quad x \in E .
$$

It is well known that $J$ is single-valued if $E^{*}$ is strictly convex. Moreover, $J(c x)=c J x$, for $\forall x \in$ $E$ and $c \in R^{1}$. We call $J$ weakly sequentially continuous if each $\left\{x_{n}\right\} \subset E$ which converges weakly to $x$ implies that $\left\{x_{n}\right\}$ converges in the sense of weak* to $J x$.

Let $C$ be a nonempty, closed, and convex subset of $E$ and $Q$ be a mapping of $E$ onto $C$. Then $Q$ is said to be sunny [1] if $Q(Q(x)+t(x-Q(x)))=Q(x)$, for all $x \in E$ and $t \geq 0$.

A mapping $Q$ of $E$ into $E$ is said to be a retraction [1] if $Q^{2}=Q$. If a mapping $Q$ is a retraction, then $Q(z)=z$ for every $z \in R(Q)$, where $R(Q)$ is the range of $Q$.

A mapping $T: C \rightarrow C$ is said to be nonexpansive if $\|T x-T y\| \leq\|x-y\|$, for $\forall x, y \in C$. We use $F(T)$ to denote the fixed point set of $T$, that is, $F(T):=\{x \in C: T x=x\}$. A mapping $T: E \supset D(T) \rightarrow R(T) \subset E$ is said to be demiclosed at $p$ if whenever $\left\{x_{n}\right\}$ is a sequence in $D(T)$ such that $x_{n} \rightarrow x \in D(T)$ and $T x_{n} \rightarrow p$ then $T x=p$.

A subset $C$ of $E$ is said to be a sunny nonexpansive retract of $E[2]$ if there exists a sunny nonexpansive retraction of $E$ onto $C$ and it is called a nonexpansive retract of $E$ if there

(c) 2014 Wei and Tan; licensee Springer. This is an Open Access article distributed under the terms of the Creative Commons Attribution License (http://creativecommons.org/licenses/by/2.0), which permits unrestricted use, distribution, and reproduction in any medium, provided the original work is properly cited. 
exists a nonexpansive retraction of $E$ onto $C$. If $E$ is reduced to a Hilbert space $H$, then the metric projection $P_{C}$ is a sunny nonexpansive retraction from $H$ to any closed and convex subset $C$ of $H$. But this is not true in a general Banach space. We note that if $E$ is smooth and $Q$ is a retraction of $C$ onto $F(T)$, then $Q$ is sunny and nonexpansive if and only if for $\forall x \in C, z \in F(T),\langle Q x-x, J(Q x-z)\rangle \leq 0[3]$.

A mapping $T: C \rightarrow C$ is called pseudo-contractive [2] if there exists $j(x-y) \in J(x-y)$ such that $\langle T x-T y, j(x-y)\rangle \leq\|x-y\|^{2}$ holds for all $x, y \in C$.

Interest in pseudo-contractive mappings stems mainly from their firm connection with the important class of nonlinear accretive mappings. A mapping $A: D(A) \subset E \rightarrow E$ is said to be accretive if $\left\|x_{1}-x_{2}\right\| \leq\left\|x_{1}-x_{2}+r\left(y_{1}-y_{2}\right)\right\|$, for $\forall x_{i} \in D(A), y_{i} \in A x_{i}, i=1,2$, and $r>0$. If $A$ is accretive, then we can define, for each $r>0$, a nonexpansive single-valued mapping $J_{r}^{A}: R(I+r A) \rightarrow D(A)$ by $J_{r}^{A}:=(I+r A)^{-1}$, which is called the resolvent of $A$. We also know that for an accretive mapping $A, N(A)=F\left(J_{r}^{A}\right)$, where $N(A)=\{x \in D(A): A x=0\}$. An accretive mapping $A$ is said to be $m$-accretive if $R(I+\lambda A)=E$, for $\forall \lambda>0$.

It is well known that if $A$ is an accretive mapping, then the solutions of the problem $0 \in A x$ correspond to the equilibrium points of some evolution equations. Hence, the problem of finding a solution $x \in E$ with $0 \in A x$ has been studied by many researchers (see [4-12] and the references contained therein).

One classical method for studying the problem $0 \in A x$, where $A$ is an $m$-accretive mapping, is the following so-called proximal method ( $c f$. [4]), presented in a Hilbert space:

$$
x_{0} \in H, \quad x_{n+1} \approx J_{r_{n}}^{A} x_{n}, \quad n \geq 0,
$$

where $J_{r_{n}}^{A}:=\left(I+r_{n} A\right)^{-1}$. It was shown that the sequence generated by (1.1) converges weakly or strongly to a zero point of $A$ under some conditions.

On the other hand, one explicit iterative process was first introduced, in 1967, by Halpern [13] in the frame of Hilbert spaces:

$$
u \in C, \quad x_{0} \in C, \quad x_{n+1}=\alpha_{n} u+\left(1-\alpha_{n}\right) T x_{n}, \quad n \geq 0,
$$

where $\left\{\alpha_{n}\right\} \subset[0,1]$ and $T: C \rightarrow C$ is a nonexpansive mapping. It was proved that under some conditions, the sequence $\left\{x_{n}\right\}$ produced by (1.2) converges strongly to a point in $F(T)$.

In 2007, Qin and Su [6] presented the following iterative algorithm:

$$
\begin{aligned}
& x_{1} \in C, \\
& y_{n}=\beta_{n} x_{n}+\left(1-\beta_{n}\right) J_{r_{n}}^{A} x_{n}, \\
& x_{n+1}=\alpha_{n} u+\left(1-\alpha_{n}\right) y_{n} .
\end{aligned}
$$

They showed that $\left\{x_{n}\right\}$ generated by (1.3) converges strongly to a point in $N(A)$.

Motivated by iterative algorithms (1.1) and (1.2), Zegeye and Shahzad extended their discussion to the case of finite $m$-accretive mappings. They presented in [14] the following iterative algorithm:

$$
x_{0} \in C, \quad x_{n+1}=\alpha_{n} u+\left(1-\alpha_{n}\right) S_{r} x_{n}, \quad n \geq 0,
$$


where $S_{r}=a_{0} I+a_{1} J_{A_{1}}+a_{2} J_{A_{2}}+\cdots+a_{l} J_{A_{l}}$ with $J_{A_{i}}=\left(I+A_{i}\right)^{-1}$ and $\sum_{i=0}^{l} a_{i}=1$. If $\bigcap_{i=1}^{l} N\left(A_{i}\right) \neq$ $\emptyset$, they proved that $\left\{x_{n}\right\}$ generated by (1.4) converges strongly to the common point in $N\left(A_{i}\right)(i=1,2, \ldots, l)$ under some conditions.

The work in [14] was then extended to the following one presented by $\mathrm{Hu}$ and $\mathrm{Liu}$ in [15]:

$$
x_{0} \in C, \quad x_{n+1}=\alpha_{n} u+\beta_{n} x_{n}+\vartheta_{n} S_{r_{n}} x_{n}, \quad n \geq 0,
$$

where $S_{r_{n}}=a_{0} I+a_{1} J_{r_{n}}^{A_{1}}+a_{2} J_{r_{n}}^{A_{2}}+\cdots+a_{l} J_{r_{n}}^{A_{l}}$ with $J_{r_{n}}^{A_{i}}=\left(I+r_{n} A_{i}\right)^{-1}$ and $\sum_{i=0}^{l} a_{i}=1$. We have $\left\{\alpha_{n}\right\},\left\{\beta_{n}\right\},\left\{\vartheta_{n}\right\} \subset(0,1)$ and $\alpha_{n}+\beta_{n}+\vartheta_{n}=1$. If $\bigcap_{i=1}^{l} N\left(A_{i}\right) \neq \emptyset$, they proved that $\left\{x_{n}\right\}$ converges strongly to the common point in $N\left(A_{i}\right)(i=1,2, \ldots, l)$ under some conditions.

In 2009, Yao et al. presented the following iterative algorithm in the frame of Hilbert space in [16]:

$$
\begin{aligned}
& x_{1} \in C, \\
& y_{n}=P_{C}\left[\left(1-\alpha_{n}\right) x_{n}\right], \\
& x_{n+1}=\left(1-\beta_{n}\right) x_{n}+\beta_{n} T y_{n}, \quad n \geq 1 .
\end{aligned}
$$

Here $T: C \rightarrow C$ is a nonexpansive mapping with $F(T) \neq \emptyset$. Suppose $\left\{\alpha_{n}\right\}$ and $\left\{\beta_{n}\right\}$ are two real sequences in $(0,1)$ satisfying

(a) $\sum_{n=1}^{\infty} \alpha_{n}=+\infty$ and $\lim _{n \rightarrow \infty} \alpha_{n}=0$;

(b) $0<\liminf _{n \rightarrow \infty} \beta_{n} \leq \limsup \sup _{n \rightarrow \infty} \beta_{n}<1$.

Then $\left\{x_{n}\right\}$ constructed by (1.6) converges strongly to a point in $F(T)$.

The following lemma is commonly used in proving the convergence of the iterative algorithms in a Banach space.

Lemma 1.1 ([17]) Let E be a real uniformly smooth Banach space, then there exists a nondecreasing continuous function $\beta:[0,+\infty) \rightarrow[0,+\infty)$ with $\lim _{t \rightarrow 0^{+}} \beta(t)=0$ and $\beta(c t) \leq$ $c \beta(t)$ for $c \geq 1$, such that for all $x, y \in E$, the following inequality holds:

$$
\|x+y\|^{2} \leq\|x\|^{2}+2\langle y, J x\rangle+\max \{\|x\|, 1\}\|y\| \beta(\|y\|)
$$

Motivated by the work in [14] and [16], and after imposing an additional condition on the function $\beta$ in Lemma 1.1 that

$$
\beta(t) \leq \frac{t}{\max \left\{1,2 r_{1}\right\}}
$$

where $r_{1}>0$ is a constant satisfying some conditions, Shehu and Ezeora presented the following result.

Theorem 1.1 ([2]) Let E be a real uniformly smooth and uniformly convex Banach space, and let $C$ be a nonempty, closed, and convex sunny nonexpansive retract of $E$, where $Q_{C}$ is the sunny nonexpansive retraction of $E$ onto $C$. Supposed the duality mapping $J: E \rightarrow E^{*}$ is weakly sequentially continuous. For each $i=1,2, \ldots, N$, let $A_{i}: C \rightarrow E$ be an m-accretive 
mapping such that $\bigcap_{i=1}^{N} N\left(A_{i}\right) \neq \emptyset$. Let $\left\{\alpha_{n}\right\},\left\{\beta_{n}\right\} \subset(0,1)$ satisfy (a) and (b). Let $\left\{x_{n}\right\}$ be generated iteratively by

$$
\begin{aligned}
& x_{1} \in C, \\
& y_{n}=Q_{C}\left[\left(1-\alpha_{n}\right) x_{n}\right], \\
& x_{n+1}=\left(1-\beta_{n}\right) x_{n}+\beta_{n} S_{N} y_{n}, \quad n \geq 1 .
\end{aligned}
$$

Here $S_{N}:=a_{0} I+a_{1} J_{A_{1}}+a_{2} J_{A_{2}}+\cdots+a_{N} J_{A_{N}}$ with $J_{A_{i}}=\left(I+A_{i}\right)^{-1}$, for $i=1,2, \ldots, N .0<a_{k}<1$, for $k=0,1,2, \ldots, N$, and $\sum_{k=0}^{N} a_{k}=1$. Then $\left\{x_{n}\right\}$ converges strongly to the common point in $N\left(A_{i}\right)$, where $i=1,2, \ldots, N$.

How do we show the convergence of the iterative sequence $\left\{x_{n}\right\}$ in (1.8) if $\beta$ loses the additional condition (1.7)? How about the convergence of $\left\{x_{n}\right\}$ if different $A_{i}$ has different coefficient in (1.8)?

To answer these questions, Wei and Tan presented the following iterative scheme in [18]:

$$
\begin{aligned}
& x_{1} \in C, \\
& u_{n}=Q_{C}\left[\left(1-\alpha_{n}\right)\left(x_{n}+e_{n}\right)\right], \\
& v_{n}=\left(1-\beta_{n}\right) x_{n}+\beta_{n} S_{n} u_{n}, \\
& x_{n+1}=\gamma_{n} x_{n}+\left(1-\gamma_{n}\right) S_{n} v_{n}, \quad n \geq 1,
\end{aligned}
$$

where $\left\{e_{n}\right\} \subset E$ is the error sequence and $\left\{A_{i}\right\}_{i=1}^{N}$ is a finite family of $m$-accretive mappings. $S_{n}:=a_{0} I+a_{1} J_{r_{n, 1}}^{A_{1}}+a_{2} J_{r_{n, 2}}^{A_{2}}+\cdots+a_{N} J_{r_{n, N}}^{A_{N}}, J_{r_{n, i}}^{A_{i}}=\left(I+r_{n, i} A_{i}\right)^{-1}$, for $i=1,2, \ldots, N, \sum_{k=0}^{N} a_{k}=1$, $0<a_{k}<1$, for $k=0,1,2, \ldots, N$. Some strong convergence theorems are obtained.

In this paper, our main purpose is to extend the discussion of (1.9) from one family of $m$-accretive mappings $\left\{A_{i}\right\}_{i=1}^{N}$ to that of two families of $m$-accretive mappings $\left\{A_{i}\right\}_{i=1}^{N}$ and $\left\{B_{j}\right\}_{j=1}^{M}$. We shall first present and study the following three-step iterative algorithm (A) with errors $\left\{e_{n}\right\} \subset E$ :

$$
\begin{aligned}
& x_{1} \in C, \\
& u_{n}=Q_{C}\left[\left(1-\alpha_{n}\right)\left(x_{n}+e_{n}\right)\right], \\
& v_{n}=\left(1-\beta_{n}\right) x_{n}+\beta_{n} S_{n} u_{n}, \\
& x_{n+1}=\gamma_{n} x_{n}+\left(1-\gamma_{n}\right) W_{n} S_{n} v_{n}, \quad n \geq 1,
\end{aligned}
$$

where $S_{n}:=a_{0} I+a_{1} J_{r_{n, 1}}^{A_{1}}+a_{2} J_{r_{n, 2}}^{A_{2}}+\cdots+a_{N} J_{r_{n, N}}^{A_{N}}$, and $W_{n}:=b_{0} I+b_{1} J_{s_{n, 1}}^{B_{1}}+b_{2} J_{s_{n, 2}}^{B_{2}}+\cdots+b_{M} J_{s_{n, M}}^{B_{M}}$. For $i=1,2, \ldots, N, J_{n, i}^{A_{i}}=\left(I+r_{n, i} A_{i}\right)^{-1}$. For $j=1,2, \ldots, M, J_{s_{n, j}}^{B_{j}}=\left(I+s_{n, j} B_{j}\right)^{-1} \cdot a_{0}, a_{1}, \ldots, a_{N}$ and $b_{0}, b_{1}, \ldots, b_{M}$ are real numbers in $(0,1)$ and $\sum_{i=0}^{N} a_{i}=1, \sum_{j=0}^{M} b_{j}=1 . r_{n, i}>0$, for $i=$ $1,2, \ldots, N$, and $s_{n, j}>0$, for $j=1,2, \ldots, M$ and $n \geq 1$.

Later, we introduce and study the following one:

$$
\begin{aligned}
& x_{1} \in C, \\
& u_{n}=Q_{C}\left[\left(1-\alpha_{n}\right)\left(x_{n}+e_{n}\right)\right],
\end{aligned}
$$




$$
\begin{aligned}
& v_{n}=\left(1-\beta_{n}\right) x_{n}+\beta_{n} S_{n} u_{n}, \\
& x_{n+1}=\gamma_{n} x_{n}+\left(1-\gamma_{n}\right) U_{n} S_{n} v_{n}, \quad n \geq 1,
\end{aligned}
$$

where $U_{n}:=c_{0} I+c_{1} J_{t_{n, 1}}^{B_{1}}+c_{2} J_{t_{n, 2}}^{B_{2}} J_{t_{n, 1}}^{B_{1}}+\cdots+c_{M} J_{t_{n, M}}^{B_{M}} J_{t_{n, M-1}}^{B_{M-1}} \ldots J_{t_{n, 1}}^{B_{1}}, c_{0}, c_{1}, \ldots, c_{M}$ are real numbers in $(0,1), \sum_{j=0}^{M} c_{j}=1$, and $J_{t_{n, j}}^{B_{j}}=\left(I+t_{n, j} B_{j}\right)^{-1}$ and $t_{n, j}>0$, for $j=1,2, \ldots, M$ and $n \geq 1$.

More details will be presented in Section 3. Some strong convergence theorems are obtained, which can be regarded as the extension of the work done in $[2,6,14,15,18]$, etc. As a consequence, some new iterative algorithms are constructed to converge strongly to the common fixed point of two finite families of pseudo-contractive mappings from $C$ to $E$.

\section{Preliminaries}

Now, we list some results we need in sequel.

Lemma 2.1 ([19]) Let E be a real uniformly convex Banach space and let $C$ be a nonempty, closed, and convex subset of $E$ and $T: C \rightarrow C$ is a nonexpansive mapping such that $F(T) \neq \emptyset$, then $I-T$ is demiclosed at zero.

Lemma 2.2 ([15]) Let E be a strictly convex Banach space which has a uniformly Gâteaux differential norm, and let $C$ be a nonempty, closed, and convex subset of E. Let $\left\{A_{i}\right\}_{i=1}^{N}$ be a finite family of accretive mappings with $\bigcap_{i=1}^{N} N\left(A_{i}\right) \neq \emptyset$, satisfying the following range conditions:

$\overline{D\left(A_{i}\right)} \subseteq C \subset \bigcap_{r>0} R\left(I+r A_{i}\right), \quad i=1,2, \ldots, N$

Let $a_{0}, a_{1}, \ldots, a_{N}$ be real numbers in $(0,1)$ such that $\sum_{i=0}^{N} a_{i}=1$ and $S_{r_{n}}=a_{0} I+a_{1} J_{r_{n}}^{A_{1}}+$ $a_{2} J_{r_{n}}^{A_{2}}+\cdots+a_{N} J_{r_{n}}^{A_{N}}$, where $J_{r_{n}}^{A_{i}}=\left(I+r_{n} A_{i}\right)^{-1}$ and $r_{n}>0$, then $S_{r_{n}}$ is nonexpansive and $F\left(S_{r_{n}}\right)=$ $\bigcap_{i=1}^{N} N\left(A_{i}\right)$.

Lemma 2.3 ([12]) In a real Banach space E, the following inequality holds:

$$
\|x+y\|^{2} \leq\|x\|^{2}+2\langle y, j(x+y)\rangle, \quad \forall x, y \in E,
$$

where $j(x+y) \in J(x+y)$.

Lemma 2.4 ([20]) Let $\left\{a_{n}\right\},\left\{b_{n}\right\}$, and $\left\{c_{n}\right\}$ be three sequences of nonnegative real numbers satisfying

$$
a_{n+1} \leq\left(1-c_{n}\right) a_{n}+b_{n} c_{n}, \quad \forall n \geq 1,
$$

where $\left\{c_{n}\right\} \subset(0,1)$ such that (i) $c_{n} \rightarrow 0$ and $\sum_{n=1}^{\infty} c_{n}=+\infty$, (ii) either lim $\sup _{n \rightarrow \infty} b_{n} \leq 0$ or $\sum_{n=1}^{\infty}\left|b_{n} c_{n}\right|<+\infty$. Then $\lim _{n \rightarrow \infty} a_{n}=0$.

Lemma 2.5 ([21]) Let $\left\{x_{n}\right\}$ and $\left\{y_{n}\right\}$ be two bounded sequences in a Banach space E such that $x_{n+1}=\beta_{n} x_{n}+\left(1-\beta_{n}\right) y_{n}$, for $n \geq 1$. Suppose $\left\{\beta_{n}\right\} \subset(0,1)$ satisfying $0<$ $\liminf _{n \rightarrow+\infty} \beta_{n} \leq \limsup _{n \rightarrow+\infty} \beta_{n}<1$. If $\lim \sup _{n \rightarrow+\infty}\left(\left\|y_{n+1}-y_{n}\right\|-\left\|x_{n+1}-x_{n}\right\|\right) \leq 0$, then $\lim _{n \rightarrow+\infty}\left\|y_{n}-x_{n}\right\|=0$. 
Lemma 2.6 ([22]) Let E be a Banach space and let A be an m-accretive mapping. For $\lambda>0$, $\mu>0$, and $x \in E$, we have

$$
J_{\lambda} x=J_{\mu}\left(\frac{\mu}{\lambda} x+\left(1-\frac{\mu}{\lambda}\right) J_{\lambda} x\right)
$$

where $J_{\lambda}=(I+\lambda A)^{-1}$ and $J_{\mu}=(I+\mu A)^{-1}$.

\section{Main results}

Lemma 3.1 ([2]) Let E be a real uniformly smooth and uniformly convex Banach space. Let $C$ be a nonempty, closed, and convex sunny nonexpansive retract of $E$, and $Q_{C}$ be the sunny nonexpansive retraction of $E$ onto $C$. Let $T: C \rightarrow C$ be nonexpansive with $F(T) \neq \emptyset$. Suppose that the duality mapping $J: E \rightarrow E^{*}$ is weakly sequentially continuous. If for each $t \in(0,1)$, define $T_{t}: C \rightarrow C$ by

$$
T_{t} x:=T Q_{C}[(1-t) x]
$$

Then $T_{t}$ is a contraction and has a fixed point $z_{t}$, which satisfies $\left\|z_{t}-T z_{t}\right\| \rightarrow 0$, as $t \rightarrow 0$.

Lemma 3.2 ([2]) Under the assumptions of Lemma 3.1, suppose further that $\beta$ in Lemma 1.1 satisfies (1.7), where $r_{1}>0$ is a sufficiently large constant such that $z_{t} \in C \bigcap\{z \in$ $\left.E:\left\|z-x^{*}\right\| \leq r_{1}\right\}, x^{*}$ is in $F(T)$ and $t \in(0,1)$, then $\lim _{t \rightarrow 0} z_{t}=z_{0} \in F(T)$.

Remark 3.1 Lemma 1.1 with additional condition (1.7) is employed as a key tool to prove Lemma 3.2. In the following lemma, we shall show that Lemma 2.3 can be used instead of Lemma 1.1, which simplifies the proof and weakens the assumption.

Lemma 3.3 Only under the assumptions of Lemma 3.1, the result of Lemma 3.2 is true, which ensures that the assumption is weaker than that in Lemma 3.2.

Proof To show that $\lim _{t \rightarrow 0} z_{t}=z_{0} \in F(T)$, it suffices to show that for any sequence $\left\{t_{n}\right\}$ such that $t_{n} \rightarrow 0$, we have $\lim _{n \rightarrow \infty} z_{t_{n}}=z_{0} \in F(T)$.

In fact, Lemma 3.1 implies that $z_{t} \in F(T)$ such that $z_{t}=T Q_{C}\left[(1-t) z_{t}\right], t \in(0,1)$. By using Lemma 2.3, we have for $\forall p \in F(T)$,

$$
\begin{aligned}
\left\|z_{t}-p\right\|^{2} & =\left\|T Q_{C}\left[(1-t) z_{t}\right]-T Q_{C} p\right\|^{2} \\
& \leq\left\|z_{t}-p-t z_{t}\right\|^{2} \\
& \leq\left\|z_{t}-p\right\|^{2}-2 t\left\|z_{t}-p-t z_{t}\right\|^{2}-2 t\left(p+t z_{t}, J\left(z_{t}-p-t z_{t}\right)\right) .
\end{aligned}
$$

This implies that

$$
\left\|z_{t}-p-t z_{t}\right\|^{2} \leq\left\langle p, J\left(p+t z_{t}-z_{t}\right)\right\rangle+t\left|z_{t}, J\left(p+t z_{t}-z_{t}\right)\right\rangle .
$$

In particular,

$$
\left\|z_{t_{n}}-p-t_{n} z_{t_{n}}\right\|^{2} \leq\left\langle p, J\left(p+t_{n} z_{t_{n}}-z_{t_{n}}\right)\right\rangle+t_{n}\left\langle z_{t_{n}}, J\left(p+t_{n} z_{t_{n}}-z_{t_{n}}\right)\right\rangle .
$$


Since $\forall p \in F(T)$,

$$
\begin{aligned}
\left\|z_{t}-p\right\| & =\left\|T Q_{C}\left[(1-t) z_{t}\right]-T Q_{C} p\right\| \\
& \leq\left\|Q_{C}\left[(1-t) z_{t}\right]-Q_{C} p\right\| \\
& \leq\left\|(1-t) z_{t}-p\right\|=\left\|(1-t)\left(z_{t}-p\right)-t p\right\| \\
& \leq(1-t)\left\|z_{t}-p\right\|+t\|p\|,
\end{aligned}
$$

$\left\{z_{t}\right\}$ is bounded.

Without loss of generality, we can assume that $\left\{z_{t_{n}}\right\}$ converges weakly to $z_{0}$. Using Lemma 3.1 and Lemma 2.1, we have $z_{0} \in F(T)$.

Substituting $z_{0}$ for $p$ in (3.3), we obtain

$$
\left\|z_{t_{n}}-z_{0}-t_{n} z_{t_{n}}\right\|^{2} \leq\left\langle z_{0}, J\left(z_{0}+t_{n} z_{t_{n}}-z_{t_{n}}\right)\right\rangle+t_{n}\left|z_{t_{n}}, J\left(z_{0}+t_{n} z_{t_{n}}-z_{t_{n}}\right)\right\rangle .
$$

Then from (3.4) and the weak convergence of $J$, we have $z_{t_{n}}-z_{0}-t_{n} z_{t_{n}} \rightarrow 0$, as $n \rightarrow \infty$.

Then from $\left\|z_{t_{n}}-z_{0}\right\| \leq\left\|z_{t_{n}}-z_{0}-t_{n} z_{t_{n}}\right\|+t_{n}\left\|z_{t_{n}}\right\|$, we see that $z_{t_{n}} \rightarrow z_{0}$, as $n \rightarrow \infty$.

Suppose there exists another sequence $z_{t_{m}} \rightarrow x_{0}$, as $t_{m} \rightarrow 0$ and $m \rightarrow \infty$. Then from Lemma 3.1 that $\left\|z_{t_{m}}-T z_{t_{m}}\right\| \rightarrow 0$ and $I-T$ is demi-closed at zero, we have $x_{0} \in F(T)$. Moreover, repeating the above proof, we have $z_{t_{m}} \rightarrow x_{0}$, as $m \rightarrow \infty$. Next, we want to show that $z_{0}=x_{0}$.

Using (3.2), we have

$$
\left\|z_{t_{m}}-z_{0}-t_{m} z_{t_{m}}\right\|^{2} \leq\left\langle z_{0}, J\left(z_{0}+t_{m} z_{t_{m}}-z_{t_{m}}\right)\right\rangle+t_{m}\left|z_{t_{m}}, J\left(z_{0}+t_{m} z_{t_{m}}-z_{t_{m}}\right)\right\rangle .
$$

By letting $m \rightarrow \infty$, (3.5) implies that

$$
\left\|x_{0}-z_{0}\right\|^{2} \leq\left\langle z_{0}, J\left(z_{0}-x_{0}\right)\right\rangle
$$

Interchanging $x_{0}$ and $z_{0}$ in (3.6), we obtain

$$
\left\|z_{0}-x_{0}\right\|^{2} \leq\left\langle x_{0}, J\left(x_{0}-z_{0}\right)\right\rangle .
$$

Then (3.6) and (3.7) ensure

$$
2\left\|x_{0}-z_{0}\right\|^{2} \leq\left\|x_{0}-z_{0}\right\|^{2},
$$

which implies that $x_{0}=z_{0}$.

Therefore, $\lim _{t \rightarrow 0} z_{t}=z_{0} \in F(T)$.

This completes the proof.

Lemma 3.4 Let $E$ be a strictly convex Banach space and let $C$ be a nonempty, closed, and convex subset of $E$. Let $A_{i}: C \rightarrow E(i=1,2, \ldots, N)$ be a finite family of m-accretive mappings such that $\bigcap_{i=1}^{N} N\left(A_{i}\right) \neq \emptyset$.

Let $a_{0}, a_{1}, \ldots, a_{N}$ be real numbers in $(0,1)$ such that $\sum_{i=0}^{N} a_{i}=1$ and $S_{n}=a_{0} I+a_{1} J_{r_{n, 1}}^{A_{1}}+$ $a_{2} J_{r_{n, 2}}^{A_{2}}+\cdots+a_{N} J_{r_{n, N}}^{A_{N}}$, where $J_{r_{n, i}}^{A_{i}}=\left(I+r_{n, i} A_{i}\right)^{-1}$ and $r_{n, i}>0$, for $i=1,2, \ldots, N$, and $n \geq 1$, then $S_{n}: C \rightarrow C$ is nonexpansive and $F\left(S_{n}\right)=\bigcap_{i=1}^{N} N\left(A_{i}\right)$, for $n \geq 1$. 
Proof The proof is from [18]. For later use, we present the proof in the following.

It is easy to check that $S_{n}: C \rightarrow C$ is nonexpansive and $\bigcap_{i=1}^{N} N\left(A_{i}\right) \subset F\left(S_{n}\right)$.

On the other hand, for $\forall p \in F\left(S_{n}\right)$, then $p=S_{n} p=a_{0} p+a_{1} J_{r_{n, 1}}^{A_{1}} p+a_{2} J_{r_{n, 2}}^{A_{2}} p+\cdots+a_{N} J_{r_{n, N}}^{A_{N}} p$. For $\forall q \in \bigcap_{i=1}^{N} N\left(A_{i}\right) \subset F\left(S_{n}\right)$, we have

$$
\begin{aligned}
\|p-q\| & \leq a_{0}\|p-q\|+a_{1}\left\|J_{r_{n, 1}}^{A_{1}} p-q\right\|+\cdots+a_{N}\left\|J_{r_{n, N}}^{A_{N}} p-q\right\| \\
& \leq\left(a_{0}+a_{1}+\cdots+a_{N-1}\right)\|p-q\|+a_{N}\left\|J_{r_{n, N}}^{A_{N}} p-q\right\| \\
& =\left(1-a_{N}\right)\|p-q\|+a_{N}\left\|J_{r_{n, N}}^{A_{N}} p-q\right\| \\
& \leq\|p-q\| .
\end{aligned}
$$

Therefore, $\|p-q\|=\left(1-a_{N}\right)\|p-q\|+a_{N}\left\|J_{r_{n, N}}^{A_{N}} p-q\right\|$, which implies that $\|p-q\|=\| J_{r_{n, N}}^{A_{N}} p-$ $q \|$. Similarly, $\|p-q\|=\left\|J_{r_{n, 1}}^{A_{1}} p-q\right\|=\cdots=\left\|J_{r_{n}, N}^{A_{N}} p-q\right\|$.

Then $\|p-q\|=\left\|\frac{a_{1}}{\sum_{i=1}^{N} a_{i}}\left(J_{r_{n, 1}}^{A_{1}} p-q\right)+\frac{a_{2}}{\sum_{i=1}^{N} a_{i}}\left(J_{r_{n}, 2}^{A_{2}} p-q\right)+\cdots+\frac{a_{N}}{\sum_{i=1}^{N} a_{i}}\left(J_{r_{n, N}}^{A_{N}} p-q\right)\right\|$, which implies from the strict convexity of $E$ that $p-q=J_{r_{1}, p}^{A_{1}} p-q=J_{r_{n, 2}}^{A_{2}} p-q=\cdots=J_{r_{n, N}}^{A_{N}} p-q$.

Therefore, $J_{r_{n, i}}^{A_{i}} p=p$, for $i=1,2, \ldots, N$. We have $p \in \bigcap_{i=1}^{N} N\left(A_{i}\right)$, which completes the proof.

Similar to Lemma 3.4, we have the following lemma.

Lemma 3.5 Let $E$ and $C$ be the same as those in Lemma 3.4. Let $\left\{B_{j}\right\}_{j=1}^{M}$ be a finite family of m-accretive mappings such that $\bigcap_{j=1}^{M} N\left(B_{j}\right) \neq \emptyset$.

Let $b_{0}, b_{1}, \ldots, b_{M}$ be real numbers in $(0,1)$ such that $\sum_{j=0}^{M} b_{j}=1$ and $W_{n}=b_{0} I+b_{1} J_{s_{n, 1}}^{B_{1}}+$ $b_{2} J_{s_{n, 2}}^{B_{2}}+\cdots+b_{M} J_{s_{n, M}}^{B_{M}}$, where $J_{s, j}^{B_{j}}=\left(I+s_{n, j} B_{j}\right)^{-1}$ and $s_{n, j}>0$, for $j=1,2, \ldots, M$, then $W_{n}: C \rightarrow C$ is nonexpansive and $F\left(W_{n}\right)=\bigcap_{j=1}^{M} N\left(B_{j}\right)$, for $n \geq 1$.

Lemma 3.6 Let E, $C, S_{n}$, and $W_{n}$ be the same as those in Lemmas 3.4 and 3.5. Suppose $D:=\left(\bigcap_{i=1}^{N} N\left(A_{i}\right)\right) \cap\left(\bigcap_{j=1}^{M} N\left(B_{j}\right)\right) \neq \emptyset$. Then $W_{n} S_{n}, S_{n} W_{n}: C \rightarrow C$ are nonexpansive and $F\left(W_{n} S_{n}\right)=F\left(S_{n} W_{n}\right)=D$.

Proof From Lemmas 3.4 and 3.5, we can easily check that $W_{n} S_{n}, S_{n} W_{n}: C \rightarrow C$ are nonexpansive and $F\left(S_{n}\right) \cap F\left(W_{n}\right)=D$. So, it suffices to show that $F\left(S_{n}\right) \cap F\left(W_{n}\right) \supset F\left(W_{n} S_{n}\right)$ since $F\left(S_{n}\right) \cap F\left(W_{n}\right) \subset F\left(W_{n} S_{n}\right)$ is trivial.

For $\forall p \in F\left(W_{n} S_{n}\right)$, then $p=W_{n} S_{n} p$.

For $\forall q \in F\left(S_{n}\right) \cap F\left(W_{n}\right) \subset F\left(W_{n} S_{n}\right)$, then $q=W_{n} S_{n} q$. Now,

$$
\|p-q\| \leq\left\|S_{n} p-S_{n} q\right\| \leq a_{0}\|p-q\|+a_{1}\left\|J_{r_{n, 1}}^{A_{1}} p-q\right\|+\cdots+a_{N}\left\|J_{r_{n, N}}^{A_{N}} p-q\right\| .
$$

Then repeating the discussion in Lemma 3.4, we know that $p \in F\left(S_{n}\right)$. Then $p=W_{n} S_{n} p=$ $W_{n} p$, thus $p \in F\left(W_{n}\right)$, which completes the proof.

Theorem 3.1 Let E be a real uniformly smooth and uniformly convex Banach space. Let $C$ be a nonempty, closed, and convex sunny nonexpansive retract of $E$, where $Q_{C}$ is the sunny nonexpansive retraction of $E$ onto $C$. Let $A_{i}, B_{j}: C \rightarrow E$ be m-accretive mappings, where $i=1,2, \ldots N, j=1,2, \ldots, M$. Suppose that the duality mapping $J: E \rightarrow E^{*}$ is weakly sequentially continuous and $D:=\left(\bigcap_{i=1}^{N} N\left(A_{i}\right)\right) \cap\left(\bigcap_{j=1}^{M} N\left(B_{j}\right)\right) \neq \emptyset$. Let $\left\{x_{n}\right\}$ be generated 
by the iterative algorithm (A), where $S_{n}:=a_{0} I+a_{1} J_{r_{n, 1}}^{A_{1}}+a_{2} J_{r_{n, 2}}^{A_{2}}+\cdots+a_{N} J_{r_{n, N}}^{A_{N}}$, and $J_{r_{n, i}}^{A_{i}}=$ $\left(I+r_{n, i} A_{i}\right)^{-1}$, for $i=1,2, \ldots, N, 0<a_{k}<1$, for $k=0,1,2, \ldots, N, \sum_{k=0}^{N} a_{k}=1 . W_{n}=b_{0} I+$ $b_{1} J_{s_{n, 1}}^{B_{1}}+b_{2} J_{s_{n, 2}}^{B_{2}}+\cdots+b_{M} J_{n_{n, M}}^{B_{M}}$, where $J_{s_{n, j}}^{B_{j}}=\left(I+s_{n, j} B_{j}\right)^{-1}$, for $j=1,2, \ldots, M, 0<b_{k}<1$, for $k=0,1,2, \ldots, M, \sum_{k=0}^{M} b_{k}=1$. Suppose $\left\{e_{n}\right\} \subset E,\left\{\alpha_{n}\right\},\left\{\beta_{n}\right\}$, and $\left\{\gamma_{n}\right\}$ are three sequences in $(0,1)$, and $\left\{r_{n, i}\right\},\left\{s_{n, j}\right\} \subset(0,+\infty)$ satisfy the following conditions:

(i) $\alpha_{n} \rightarrow 0, \beta_{n} \rightarrow 0$, as $n \rightarrow \infty$;

(ii) $\sum_{n=1}^{\infty} \alpha_{n} \beta_{n}=+\infty$;

(iii) $0<\liminf _{n \rightarrow+\infty} \gamma_{n} \leq \limsup _{n \rightarrow+\infty} \gamma_{n}<1$;

(iv) $\sum_{n=1}^{\infty}\left|r_{n+1, i}-r_{n, i}\right|<+\infty$ and $r_{n, i} \geq \varepsilon>0$, for $n \geq 1$ and $i=1,2, \ldots, N$;

(v) $\sum_{n=1}^{\infty}\left|s_{n+1, j}-s_{n, j}\right|<+\infty$ and $s_{n, j} \geq \varepsilon>0$, for $n \geq 1$ and $j=1,2, \ldots, M$;

(vi) $\frac{\left\|e_{n}\right\|}{\alpha_{n}} \rightarrow 0$, as $n \rightarrow+\infty$, and $\sum_{n=1}^{\infty}\left\|e_{n}\right\|<+\infty$.

Then $\left\{x_{n}\right\}$ converges strongly to a point $p_{0} \in D$.

Proof We shall split the proof into five steps:

Step 1. $\left\{x_{n}\right\},\left\{u_{n}\right\},\left\{S_{n} u_{n}\right\},\left\{v_{n}\right\}$, and $\left\{S_{n} x_{n}\right\}$ are all bounded.

We shall first show that $\forall p \in D$,

$$
\left\|x_{n+1}-p\right\| \leq M_{1}+\sum_{i=1}^{n}\left\|e_{i}\right\|,
$$

where $M_{1}=\max \left\{\left\|x_{1}-p\right\|,\|p\|\right\}$.

By using the induction method, we see that for $n=1, \forall p \in D$,

$$
\begin{aligned}
\left\|x_{2}-p\right\| & \leq \gamma_{1}\left\|x_{1}-p\right\|+\left(1-\gamma_{1}\right)\left\|W_{1} S_{1} v_{1}-p\right\| \\
& \leq \gamma_{1}\left\|x_{1}-p\right\|+\left(1-\gamma_{1}\right)\left\|v_{1}-p\right\| \\
& \leq \gamma_{1}\left\|x_{1}-p\right\|+\left(1-\gamma_{1}\right)\left(1-\beta_{1}\right)\left\|x_{1}-p\right\|+\beta_{1}\left(1-\gamma_{1}\right)\left\|u_{1}-p\right\| \\
& \leq \gamma_{1}\left\|x_{1}-p\right\|+\left(1-\gamma_{1}\right)\left(1-\beta_{1}\right)\left\|x_{1}-p\right\|+\beta_{1}\left(1-\gamma_{1}\right)\left\|\left(1-\alpha_{1}\right)\left(x_{1}+e_{1}\right)-p\right\| \\
& \leq\left[1-\alpha_{1} \beta_{1}\left(1-\gamma_{1}\right)\right]\left\|x_{1}-p\right\|+\alpha_{1} \beta_{1}\left(1-\gamma_{1}\right)\|p\|+\left(1-\alpha_{1}\right) \beta_{1}\left(1-\gamma_{1}\right)\left\|e_{1}\right\| \\
& \leq M_{1}+\left\|e_{1}\right\| .
\end{aligned}
$$

Suppose that (3.9) is true for $n=k$. Then, for $n=k+1$,

$$
\begin{aligned}
\left\|x_{k+2}-p\right\| \leq & \gamma_{k+1}\left\|x_{k+1}-p\right\|+\left(1-\gamma_{k+1}\right)\left\|v_{k+1}-p\right\| \\
\leq & \gamma_{k+1}\left\|x_{k+1}-p\right\|+\left(1-\gamma_{k+1}\right)\left[\left(1-\beta_{k+1}\right)\left\|x_{k+1}-p\right\|+\beta_{k+1}\left\|u_{k+1}-p\right\|\right] \\
\leq & \gamma_{k+1}\left\|x_{k+1}-p\right\|+\left(1-\gamma_{k+1}\right)\left[\left(1-\beta_{k+1}\right)\left\|x_{k+1}-p\right\|\right. \\
& \left.\quad+\beta_{k+1}\left\|\left(1-\alpha_{k+1}\right)\left(x_{k+1}+e_{k+1}\right)-p\right\|\right] \\
\leq & {\left[1-\alpha_{k+1} \beta_{k+1}\left(1-\gamma_{k+1}\right)\right]\left\|x_{k+1}-p\right\|+\alpha_{k+1} \beta_{k+1}\left(1-\gamma_{k+1}\right)\|p\| } \\
& \quad+\beta_{k+1}\left(1-\alpha_{k+1}\right)\left(1-\gamma_{k+1}\right)\left\|e_{k+1}\right\| \\
\leq & M_{1}+\left[1-\alpha_{k+1} \beta_{k+1}\left(1-\gamma_{k+1}\right)\right] \sum_{i=1}^{k}\left\|e_{i}\right\|+\left(1-\alpha_{k+1}\right) \beta_{k+1}\left(1-\gamma_{k+1}\right)\left\|e_{k+1}\right\| \\
\leq & M_{1}+\sum_{i=1}^{k+1}\left\|e_{i}\right\| .
\end{aligned}
$$


Thus (3.9) is true for all $n \in N$. Since $\sum_{n=1}^{\infty}\left\|e_{n}\right\|<+\infty$, (3.9) ensures that $\left\{x_{n}\right\}$ is bounded. For $\forall p \in D$, from $\left\|u_{n}-p\right\| \leq\left\|\left(1-\alpha_{n}\right)\left(x_{n}+e_{n}\right)-p\right\| \leq\left\|x_{n}\right\|+\left\|e_{n}\right\|+\|p\|$, we see that $\left\{u_{n}\right\}$ is bounded.

Since $\left\|S_{n} u_{n}\right\| \leq\left\|S_{n} u_{n}-S_{n} p\right\|+\|p\| \leq\left\|u_{n}-p\right\|+\|p\|,\left\{S_{n} u_{n}\right\}$ is bounded. Since both $\left\{S_{n} u_{n}\right\}$ and $\left\{x_{n}\right\}$ are bounded, $\left\{v_{n}\right\}$ is bounded. Similarly, $\left\{S_{n} x_{n}\right\},\left\{S_{n} v_{n}\right\},\left\{J_{r_{n, i}}^{A_{i}} u_{n}\right\},\left\{J_{r_{n, i}}^{A_{i}} v_{n}\right\}$, and $\left\{s_{s_{n, j}}^{B_{j}} S_{n} v_{n}\right\}$ are all bounded, for $i=1,2, \ldots, N ; j=1,2, \ldots, M$.

Then we set $M_{2}=\sup \left\{\left\|u_{n}\right\|,\left\|J_{r_{n, i}}^{A_{i}} u_{n}\right\|,\left\|v_{n}\right\|,\left\|J_{r_{n, i}}^{A_{i}} v_{n}\right\|,\left\|S_{n} u_{n}\right\|,\left\|S_{n} v_{n}\right\|,\left\|x_{n}\right\|,\left\|J_{s_{n, j}}^{B_{j}} S_{n} v_{n}\right\|: n \geq\right.$ $1, i=1,2, \ldots, N ; j=1,2, \ldots, M\}$.

Step 2. $\lim _{n \rightarrow \infty}\left\|x_{n}-W_{n} S_{n} v_{n}\right\|=0$ and $\lim _{n \rightarrow \infty}\left\|x_{n+1}-x_{n}\right\|=0$.

In fact,

$$
\begin{aligned}
& \left\|W_{n+1} S_{n+1} v_{n+1}-W_{n} S_{n} v_{n}\right\| \\
& \quad \leq b_{0}\left\|S_{n+1} v_{n+1}-S_{n} v_{n}\right\|+\sum_{j=1}^{M} b_{j}\left\|J_{s_{n+1, j}}^{B_{j}} S_{n+1} v_{n+1}-J_{n_{n, j}}^{B_{j}} S_{n} v_{n}\right\| .
\end{aligned}
$$

Next, we discuss $\left\|J_{s_{n+1, j}}^{B_{j}} S_{n+1} v_{n+1}-\int_{s_{n, j}}^{B_{j}} S_{n} v_{n}\right\|$.

If $s_{n, j} \leq s_{n+1, j}$, then, using Lemma 2.6,

$$
\begin{aligned}
& \left\|J_{s_{n+1, j}}^{B_{j}} S_{n+1} v_{n+1}-J_{s_{n, j}}^{B_{j}} S_{n} v_{n}\right\| \\
& \quad=\left\|J_{n_{n, j}}^{B_{j}}\left(\frac{s_{n, j}}{s_{n+1, j}} S_{n+1} v_{n+1}+\left(1-\frac{s_{n, j}}{s_{n+1, j}}\right) J_{s_{n+1, j}}^{B_{j}} S_{n+1} v_{n+1}\right)-J_{s_{n, j}}^{B_{j}} S_{n} v_{n}\right\| \\
& \quad \leq\left\|\frac{s_{n, j}}{s_{n+1, j}} S_{n+1} v_{n+1}+\left(1-\frac{s_{n, j}}{s_{n+1, j}}\right) J_{s_{n+1, j}}^{B_{j}} S_{n+1} v_{n+1}-S_{n} v_{n}\right\| \\
& \quad \leq \frac{s_{n, j}}{s_{n+1, j}}\left\|S_{n+1} v_{n+1}-S_{n} v_{n}\right\|+\left(1-\frac{s_{n, j}}{s_{n+1, j}}\right)\left\|J_{s_{n+1, j}}^{B_{j}} S_{n+1} v_{n+1}-S_{n} v_{n}\right\| \\
& \quad \leq\left\|S_{n+1} v_{n+1}-S_{n} v_{n}\right\|+2 M_{2} \frac{s_{n+1, j}-s_{n, j}}{\varepsilon} .
\end{aligned}
$$

If $s_{n+1, j} \leq s_{n, j}$, then imitating the proof of (3.11), we have

$$
\left\|J_{s_{n+1, j}}^{B_{j}} S_{n+1} v_{n+1}-J_{s_{n, j}}^{B_{j}} S_{n} v_{n}\right\| \leq\left\|S_{n+1} v_{n+1}-S_{n} v_{n}\right\|+2 M_{2} \frac{S_{n, j}-s_{n+1, j}}{\varepsilon} .
$$

Combining (3.11) and (3.12), we have

$$
\begin{aligned}
& \left\|J_{s_{n+1, j}}^{B_{j}} S_{n+1} v_{n+1}-J_{s_{n, j}}^{B_{j}} S_{n} v_{n}\right\| \\
& \quad \leq\left\|S_{n+1} v_{n+1}-S_{n} v_{n}\right\|+2 M_{2} \frac{\left|s_{n, j}-s_{n+1, j}\right|}{\varepsilon} .
\end{aligned}
$$

Putting (3.13) into (3.10), we have

$$
\left\|W_{n+1} S_{n+1} v_{n+1}-W_{n} S_{n} v_{n}\right\| \leq\left\|S_{n+1} v_{n+1}-S_{n} v_{n}\right\|+\frac{2 M_{2}}{\varepsilon} \sum_{j=1}^{M}\left|s_{n, j}-s_{n+1, j}\right| .
$$


Similarly, we have

$$
\left\|S_{n+1} u_{n+1}-S_{n} u_{n}\right\| \leq\left\|u_{n+1}-u_{n}\right\|+\frac{2 M_{2}}{\varepsilon} \sum_{i=1}^{N}\left|r_{n, i}-r_{n+1, i}\right|
$$

and

$$
\left\|S_{n+1} v_{n+1}-S_{n} v_{n}\right\| \leq\left\|v_{n+1}-v_{n}\right\|+\frac{2 M_{2}}{\varepsilon} \sum_{i=1}^{N}\left|r_{n, i}-r_{n+1, i}\right| .
$$

Therefore,

$$
\begin{aligned}
& \left\|W_{n+1} S_{n+1} v_{n+1}-W_{n} S_{n} v_{n}\right\| \\
& \leq\left\|v_{n+1}-v_{n}\right\|+\frac{2 M_{2}}{\varepsilon} \sum_{j=1}^{M}\left|s_{n, j}-s_{n+1, j}\right|+\frac{2 M_{2}}{\varepsilon} \sum_{i=1}^{N}\left|r_{n, i}-r_{n+1, i}\right| \\
& \leq\left\|x_{n+1}-x_{n}\right\|+\beta_{n}\left\|x_{n}\right\|+\beta_{n+1}\left\|x_{n+1}\right\|+\left|\beta_{n+1}-\beta_{n}\right|\left\|S_{n+1} u_{n+1}\right\|+\beta_{n}\left\|S_{n+1} u_{n+1}-S_{n} u_{n}\right\| \\
& +\frac{2 M_{2}}{\varepsilon} \sum_{j=1}^{M}\left|s_{n, j}-s_{n+1, j}\right|+\frac{2 M_{2}}{\varepsilon} \sum_{i=1}^{N}\left|r_{n, i}-r_{n+1, i}\right| \\
& \leq\left\|x_{n+1}-x_{n}\right\|+\beta_{n}\left\|x_{n}\right\|+\beta_{n+1}\left\|x_{n+1}\right\|+\left|\beta_{n+1}-\beta_{n}\right|\left\|S_{n+1} u_{n+1}\right\|+\beta_{n}\left\|u_{n+1}-u_{n}\right\| \\
& +\frac{4 M_{2}}{\varepsilon} \sum_{i=1}^{N}\left|r_{n, i}-r_{n+1, i}\right|+\frac{2 M_{2}}{\varepsilon} \sum_{j=1}^{M}\left|s_{n, j}-s_{n+1, j}\right| \\
& \leq\left\|x_{n+1}-x_{n}\right\|+\beta_{n}\left\|x_{n}\right\|+\beta_{n+1}\left\|x_{n+1}\right\|+\left|\beta_{n+1}-\beta_{n}\right|\left\|S_{n+1} u_{n+1}\right\| \\
& +\beta_{n}\left\|\left(1-\alpha_{n+1}\right)\left(x_{n+1}+e_{n+1}\right)-\left(1-\alpha_{n}\right)\left(x_{n}+e_{n}\right)\right\| \\
& +\frac{4 M_{2}}{\varepsilon} \sum_{i=1}^{N}\left|r_{n, i}-r_{n+1, i}\right|+\frac{2 M_{2}}{\varepsilon} \sum_{j=1}^{M}\left|s_{n, j}-s_{n+1, j}\right| \\
& \leq\left(1+\beta_{n}\right)\left\|x_{n+1}-x_{n}\right\|+\left(\beta_{n}+\alpha_{n} \beta_{n}\right)\left\|x_{n}\right\|+\left(\beta_{n+1}+\alpha_{n+1} \beta_{n}\right)\left\|x_{n+1}\right\| \\
& +\left|\beta_{n+1}-\beta_{n}\right|\left\|S_{n+1} u_{n+1}\right\|+\beta_{n}\left\|e_{n+1}-e_{n}\right\|+\beta_{n}\left\|\alpha_{n+1} e_{n+1}-\alpha_{n} e_{n}\right\| \\
& +\frac{4 M_{2}}{\varepsilon} \sum_{i=1}^{N}\left|r_{n, i}-r_{n+1, i}\right|+\frac{2 M_{2}}{\varepsilon} \sum_{j=1}^{M}\left|s_{n, j}-s_{n+1, j}\right| \text {. }
\end{aligned}
$$

Thus limsup $\sup _{n \rightarrow+\infty}\left(\left\|W_{n+1} S_{n+1} v_{n+1}-W_{n} S_{n} v_{n}\right\|-\left\|x_{n+1}-x_{n}\right\|\right) \leq 0$. Using Lemma 2.5, we have from (3.17) $\lim _{n \rightarrow \infty}\left\|x_{n}-W_{n} S_{n} v_{n}\right\|=0$ and then $\lim _{n \rightarrow \infty}\left\|x_{n+1}-x_{n}\right\|=\lim _{n \rightarrow \infty}(1-$ $\left.\gamma_{n}\right)\left\|W_{n} S_{n} v_{n}-x_{n}\right\|=0$

Step 3. $\lim _{n \rightarrow \infty}\left\|x_{n}-W_{n} S_{n} x_{n}\right\|=0$.

In fact,

$$
\begin{aligned}
\left\|x_{n}-W_{n} S_{n} x_{n}\right\| & \leq\left\|x_{n+1}-x_{n}\right\|+\left\|x_{n+1}-W_{n} S_{n} x_{n}\right\| \\
& \leq\left\|x_{n+1}-x_{n}\right\|+\left\|\gamma_{n} x_{n}+\left(1-\gamma_{n}\right) W_{n} S_{n} v_{n}-W_{n} S_{n} x_{n}\right\| \\
& \leq\left\|x_{n+1}-x_{n}\right\|+\gamma_{n}\left\|x_{n}-W_{n} S_{n} v_{n}\right\|+\left\|W_{n} S_{n} v_{n}-W_{n} S_{n} x_{n}\right\|
\end{aligned}
$$




$$
\begin{aligned}
& \leq\left\|x_{n+1}-x_{n}\right\|+\gamma_{n}\left\|x_{n}-W_{n} S_{n} v_{n}\right\|+\beta_{n}\left\|S_{n} u_{n}-x_{n}\right\| \\
& \leq\left\|x_{n+1}-x_{n}\right\|+\gamma_{n}\left\|x_{n}-W_{n} S_{n} v_{n}\right\|+2 \beta_{n} M_{2} .
\end{aligned}
$$

Then (3.18) and step 2 imply that $\left\|x_{n}-W_{n} S_{n} x_{n}\right\| \rightarrow 0$, as $n \rightarrow+\infty$, since $\beta_{n} \rightarrow 0$.

Step 4. $\limsup _{n \rightarrow+\infty}\left\langle p_{0}, J\left(p_{0}-x_{n}\right)\right\rangle \leq 0$, where $p_{0}$ is an element in $D$.

From Lemma 3.6, we know that $W_{n} S_{n}: C \rightarrow C$ is nonexpansive and $F\left(W_{n} S_{n}\right)=D$. Then Lemma 3.1 and Lemma 3.3 imply that there exists $z_{t} \in C$ such that $z_{t}=W_{n} S_{n} Q_{C}\left[(1-t) z_{t}\right]$ for $t \in(0,1)$. Moreover, $z_{t} \rightarrow p_{0} \in D$, as $t \rightarrow 0$.

Since $\left\|z_{t}-p_{0}\right\| \leq\left\|(1-t) z_{t}-p_{0}\right\| \leq(1-t)\left\|z_{t}-p_{0}\right\|+\left\|p_{0}\right\|,\left\{z_{t}\right\}$ is bounded. Let $M_{3}=$ $\sup \left\{\left\|z_{t}-x_{n}\right\|: n \geq 1, t>0\right\}$. Then from step 1 , we know that $M_{3}$ is a positive constant. Using Lemma 2.3, we have

$$
\begin{aligned}
\left\|z_{t}-x_{n}\right\|^{2} & =\left\|z_{t}-W_{n} S_{n} x_{n}+W_{n} S_{n} x_{n}-x_{n}\right\|^{2} \\
& \leq\left\|z_{t}-W_{n} S_{n} x_{n}\right\|^{2}+2\left\langle W_{n} S_{n} x_{n}-x_{n}, J\left(z_{t}-x_{n}\right)\right\rangle \\
& \leq\left\|z_{t}-W_{n} S_{n} x_{n}\right\|^{2}+2\left\|W_{n} S_{n} x_{n}-x_{n}\right\|\left\|z_{t}-x_{n}\right\| \\
& \leq\left\|(1-t) z_{t}-x_{n}\right\|^{2}+2\left\|W_{n} S_{n} x_{n}-x_{n}\right\|\left\|z_{t}-x_{n}\right\| \\
& \leq\left\|z_{t}-x_{n}\right\|^{2}-2 t\left(z_{t}, J\left[(1-t) z_{t}-x_{n}\right]\right\rangle+2 M_{3}\left\|W_{n} S_{n} x_{n}-x_{n}\right\| .
\end{aligned}
$$

So $\left\langle z_{t}, J\left[(1-t) z_{t}-x_{n}\right]\right\rangle \leq \frac{M_{3}}{t}\left\|W_{n} S_{n} x_{n}-x_{n}\right\|$, which implies that $\lim _{t \rightarrow 0} \lim \sup _{n \rightarrow+\infty}\left\langle z_{t}\right.$, $\left.J\left[(1-t) z_{t}-x_{n}\right]\right\rangle \leq 0$ in view of step 3 .

Since $\left\{x_{n}\right\}$ is bounded and $J$ is uniformly continuous on each bounded subset of $E$, $\left\langle p_{0}, J\left(p_{0}-x_{n}\right)-J\left[(1-t) z_{t}-x_{n}\right]\right\rangle \rightarrow 0$, as $t \rightarrow 0$.

Moreover, noticing the fact that

$$
\begin{aligned}
\left\langle p_{0}, J\left(p_{0}-x_{n}\right)\right\rangle= & \left\langle p_{0}, J\left(p_{0}-x_{n}\right)-J\left[(1-t) z_{t}-x_{n}\right]\right\rangle \\
& +\left\langle p_{0}-z_{t}, J\left[(1-t) z_{t}-x_{n}\right]\right\rangle+\left\langle z_{t}, J\left[(1-t) z_{t}-x_{n}\right]\right\rangle,
\end{aligned}
$$

we have $\lim \sup _{n \rightarrow+\infty}\left\langle p_{0}, J\left(p_{0}-x_{n}\right)\right\rangle \leq 0$.

Since $\left\langle p_{0}, J\left[p_{0}-x_{n}-\left(1-\alpha_{n}\right) e_{n}+\alpha_{n} x_{n}\right]\right\rangle=\left\langle p_{0}, J\left[p_{0}-x_{n}-\left(1-\alpha_{n}\right) e_{n}+\alpha_{n} x_{n}\right]-J\left(p_{0}-x_{n}\right)\right\rangle+$ $\left\langle p_{0}, J\left(p_{0}-x_{n}\right)\right\rangle$, and $J$ is uniformly continuous on each bounded subset of $E$,

$$
\limsup _{n \rightarrow+\infty}\left\langle p_{0}, J\left[p_{0}-x_{n}-\left(1-\alpha_{n}\right) e_{n}+\alpha_{n} x_{n}\right]\right\rangle \leq 0
$$

Step 5. $x_{n} \rightarrow p_{0}$, as $n \rightarrow+\infty$, where $p_{0} \in D$ is the same as in step 4 .

Let $M_{4}=\sup \left\{\left\|\left(1-\alpha_{n}\right)\left(x_{n}+e_{n}\right)-p_{0}\right\|: n \geq 1\right\}$. By using Lemma 2.3 again, we have

$$
\begin{aligned}
& \left\|x_{n+1}-p_{0}\right\|^{2} \\
& \quad \leq \gamma_{n}\left\|x_{n}-p_{0}\right\|^{2}+\left(1-\gamma_{n}\right)\left\|v_{n}-p_{0}\right\|^{2} \\
& \quad \leq \gamma_{n}\left\|x_{n}-p_{0}\right\|^{2}+\left(1-\gamma_{n}\right)\left(1-\beta_{n}\right)\left\|x_{n}-p_{0}\right\|^{2}+\left(1-\gamma_{n}\right) \beta_{n}\left\|u_{n}-p_{0}\right\|^{2} \\
& \quad=\left(1-\beta_{n}+\beta_{n} \gamma_{n}\right)\left\|x_{n}-p_{0}\right\|^{2}+\left(1-\gamma_{n}\right) \beta_{n}\left\|u_{n}-p_{0}\right\|^{2} \\
& \quad \leq\left(1-\beta_{n}+\beta_{n} \gamma_{n}\right)\left\|x_{n}-p_{0}\right\|^{2}+\left(1-\gamma_{n}\right) \beta_{n}\left\|\left(1-\alpha_{n}\right)\left(x_{n}+e_{n}\right)-p_{0}\right\|^{2}
\end{aligned}
$$




$$
\begin{aligned}
\leq & \left(1-\beta_{n}+\beta_{n} \gamma_{n}\right)\left\|x_{n}-p_{0}\right\|^{2}+\left(1-\gamma_{n}\right) \beta_{n}\left(1-\alpha_{n}\right)\left\|x_{n}-p_{0}\right\|^{2} \\
& +2\left(1-\gamma_{n}\right) \beta_{n}\left(1-\alpha_{n}\right)\left\langle e_{n}, J\left[\left(1-\alpha_{n}\right)\left(x_{n}+e_{n}\right)-p_{0}\right]\right\rangle \\
& +2 \alpha_{n} \beta_{n}\left(1-\gamma_{n}\right)\left(p_{0}, J\left[p_{0}-x_{n}-\left(1-\alpha_{n}\right) e_{n}+\alpha_{n} x_{n}\right]\right\rangle \\
\leq & {\left[1-\alpha_{n} \beta_{n}\left(1-\gamma_{n}\right)\right]\left\|x_{n}-p_{0}\right\|^{2}+2\left(1-\gamma_{n}\right)\left(1-\alpha_{n}\right) \beta_{n} M_{4}\left\|e_{n}\right\| } \\
& +2 \alpha_{n} \beta_{n}\left(1-\gamma_{n}\right)\left\langle p_{0}, J\left[p_{0}-x_{n}-\left(1-\alpha_{n}\right) e_{n}+\alpha_{n} x_{n}\right]\right\rangle .
\end{aligned}
$$

Let $c_{n}=\left(1-\gamma_{n}\right) \alpha_{n} \beta_{n}$, then (3.20) reduces to $\left\|x_{n+1}-p_{0}\right\|^{2} \leq\left(1-c_{n}\right)\left\|x_{n}-p_{0}\right\|^{2}+$ $2 c_{n}\left\{\left\langle p_{0}, J\left[p_{0}-x_{n}-\left(1-\alpha_{n}\right) e_{n}+\alpha_{n} x_{n}\right]\right\rangle+\left(1-\alpha_{n}\right) M_{4} \frac{\left\|e_{n}\right\|}{\alpha_{n}}\right\}$.

From (3.19), (3.20), and the assumptions, by using Lemma 2.4, we know that $x_{n} \rightarrow p_{0}$, as $n \rightarrow+\infty$.

This completes the proof.

If in Theorem 3.1, $C=E$, then we have the following theorem.

Theorem 3.2 Let $E$ and $D$ be the same as those in Theorem 3.1. Suppose that the duality mapping $J: E \rightarrow E^{*}$ is weakly sequentially continuous. Let $A_{i}: E \rightarrow E(i=1,2, \ldots, N)$ and $B_{j}: E \rightarrow E(j=1,2, \ldots, M)$ be two finite families of m-accretive mappings. Let $\left\{e_{n}\right\} \subset E$, $\left\{\alpha_{n}\right\},\left\{\beta_{n}\right\},\left\{\gamma_{n}\right\} \subset(0,1)$, and $\left\{r_{n, i}\right\},\left\{s_{n, j}\right\} \subset(0,+\infty)$ satisfy the some conditions presented in Theorem 3.1.

Let $\left\{x_{n}\right\}$ be generated by the following scheme:

$$
\begin{aligned}
& x_{1} \in E, \\
& u_{n}=\left(1-\alpha_{n}\right)\left(x_{n}+e_{n}\right), \\
& v_{n}=\left(1-\beta_{n}\right) x_{n}+\beta_{n} S_{n} u_{n}, \\
& x_{n+1}=\gamma_{n} x_{n}+\left(1-\gamma_{n}\right) W_{n} S_{n} v_{n}, \quad n \geq 1 .
\end{aligned}
$$

Then $\left\{x_{n}\right\}$ converges strongly to a point $p_{0} \in D$, where $S_{n}$ and $W_{n}$ are the same as those in Theorem 3.1.

Lemma 3.7 Let E, $C$ and $\left\{B_{j}\right\}_{j=1}^{M}$ be the same as those in Lemma 3.5. $\bigcap_{j=1}^{M} N\left(B_{j}\right) \neq \emptyset$.

Let $c_{0}, c_{1}, \ldots, c_{M}$ be real numbers in $(0,1)$ such that $\sum_{j=0}^{M} c_{j}=1$ and $U_{n}=c_{0} I+c_{1} J_{t_{n, 1}}^{B_{1}}+$ $c_{2} J_{t_{n, 2}}^{B_{2}} J_{t_{n, 1}}^{B_{1}}+\cdots+c_{M} J_{t_{n, M}}^{B_{M}} J_{t_{n, M-1}}^{B_{M-1}} \cdots J_{t_{n, 1}}^{B_{1}}$, where $J_{t_{n, j}}^{B_{j}}=\left(I+t_{n, j} B_{j}\right)^{-1}$ and $t_{n, j}>0$, for $j=1,2, \ldots, M$, and $n \geq 1$, then $U_{n}: C \rightarrow C$ is nonexpansive and $F\left(U_{n}\right)=\bigcap_{j=1}^{M} N\left(B_{j}\right)$, for $n \geq 1$.

Proof It is easy to check that $U_{n}: C \rightarrow C$ is nonexpansive and $\bigcap_{j=1}^{M} N\left(B_{j}\right) \subset F\left(U_{n}\right)$.

On the other hand, for $\forall p \in F\left(U_{n}\right)$, then $p=U_{n} p=c_{0} p+c_{1} J_{t_{n, 1}}^{B_{1}} p+c_{2} J_{t_{n, 2}}^{B_{2}} J_{t_{n, 1}}^{B_{1}} p+\cdots+$ $c_{M} J_{t_{n, M}}^{B_{M}} J_{t_{n, M-1}}^{B_{M-1}} \cdots J_{t_{n, 1}}^{B_{1}} p$.

For $\forall q \in \bigcap_{j=1}^{M} N\left(B_{j}\right) \subset F\left(U_{n}\right)$, then

$$
\begin{aligned}
\|p-q\| & \leq c_{0}\|p-q\|+c_{1}\left\|J_{t_{n, 1}}^{B_{1}} p-q\right\|+\cdots+c_{M}\left\|J_{t_{n, M}}^{B_{M}} J_{t_{n, M-1}}^{B_{M-1}} \cdots J_{t_{n, 1}}^{B_{1}} p-q\right\| \\
& \leq\left(c_{0}+c_{2}+\cdots+c_{M}\right)\|p-q\|+c_{1}\left\|J_{t_{n, 1}}^{B_{1}} p-q\right\| \\
& =\left(1-c_{1}\right)\|p-q\|+c_{1}\left\|J_{t_{n, 1}}^{B_{1}} p-q\right\| \\
& \leq\|p-q\| .
\end{aligned}
$$


Therefore, $\|p-q\|=\left(1-c_{1}\right)\|p-q\|+c_{1}\left\|J_{t_{n, 1}}^{B_{1}} p-q\right\|$, which implies that $\|p-q\|=\left\|J_{t_{n, 1}}^{B_{1}} p-q\right\|$. Similarly, $\|p-q\|=\left\|J_{t_{n, 1}}^{B_{1}} p-q\right\|=\left\|J_{t_{n, 2}}^{B_{2}} J_{t_{n, 1}}^{B_{1}} p-q\right\|=\cdots=\left\|J_{t_{n, M}}^{B_{M}} J_{t_{n, M-1}}^{B_{M-1}} \cdots J_{t_{n, 1}}^{B_{1}} p-q\right\|$.

Then $\|p-q\|=\| \frac{c_{1}}{\sum_{j=1}^{M} c_{j}}\left(J_{t_{n},}^{B_{1}} p-q\right)+\frac{c_{2}}{\sum_{j=1}^{M} c_{j}}\left(J_{t_{n, 2}}^{B_{2}} J_{t_{n, 1}}^{B_{1}} p-q\right)+\cdots+\frac{c_{M}}{\sum_{j=1}^{M} c_{j}} J_{t_{n, M}}^{B_{M}} J_{t_{n, M-1}}^{B_{M-1}} \cdots J_{t_{n, 1}}^{B_{1}} p-$ $q) \|$, which implies from the strict convexity of $E$ that $p-q=J_{t_{n, 1}}^{B_{1}} p-q=J_{t_{n, 2}}^{B_{2}} J_{t_{n, 1}}^{B_{1}} p-q=\cdots=$ $J_{t_{n, M}, J_{M, M-1}}^{B_{M}} J_{t_{n-1}}^{B_{M-1}} J_{t_{n, 1}}^{B_{1}} p-q$.

Therefore, $J_{t_{n, 1}}^{B_{1}} p=p$, and then we can easily see that $J_{t_{n, j}}^{B_{j}} p=p$, for $j=2, \ldots, M$. Thus $p \in$ $\bigcap_{j=1}^{M} N\left(B_{j}\right)$, which completes the proof.

Lemma 3.8 Let $E$ and $C$ be the same as those in Lemma 3.4. Let $S_{n}$ and $U_{n}$ be the same as those in Lemmas 3.4 and 3.7, respectively. Suppose $D:=\left(\bigcap_{i=1}^{N} N\left(A_{i}\right)\right) \cap\left(\bigcap_{j=1}^{M} N\left(B_{j}\right)\right) \neq \emptyset$. Then $S_{n} U_{n}, U_{n} S_{n}: C \rightarrow C$ are nonexpansive and $F\left(U_{n} S_{n}\right)=F\left(S_{n} U_{n}\right)=D$.

Proof From Lemmas 3.4 and 3.7, we can easily check that $U_{n} S_{n}, S_{n} U_{n}: C \rightarrow C$ are nonexpansive and $F\left(S_{n}\right) \cap F\left(U_{n}\right)=D$. So, it suffices to show that $F\left(S_{n}\right) \cap F\left(U_{n}\right) \supset F\left(U_{n} S_{n}\right)$ since $F\left(S_{n}\right) \cap F\left(U_{n}\right) \subset F\left(U_{n} S_{n}\right)$ is trivial.

For $\forall p \in F\left(U_{n} S_{n}\right)$, then $p=U_{n} S_{n} p$.

For $\forall q \in F\left(S_{n}\right) \cap F\left(U_{n}\right) \subset F\left(U_{n} S_{n}\right)$, then $q=U_{n} S_{n} q$. Now,

$$
\|p-q\|=\left\|U_{n} S_{n} p-q\right\| \leq\left\|S_{n} p-S_{n} q\right\| \leq\|p-q\| .
$$

Then repeating the discussion in Lemma 3.4, we know that $p \in F\left(S_{n}\right)$. Then $p=U_{n} S_{n} p=$ $U_{n} p$, thus $p \in F\left(U_{n}\right)$, which completes the proof.

Theorem 3.3 Let E, $C, Q_{C}, S_{n}$, and $D$ be the same as those in Theorem 3.1. Let $A_{i}, B_{j}: C \rightarrow$ $E$ be m-accretive mappings, for $i=1,2, \ldots N$, and $j=1,2, \ldots, M$. Suppose that the duality mapping $J: E \rightarrow E^{*}$ is weakly sequentially continuous and $D \neq \emptyset$. Let $\left\{x_{n}\right\}$ be generated by the iterative algorithm (B), where $U_{n}:=c_{0} I+c_{1} J_{t_{n, 1}}^{B_{1}}+c_{2} J_{t_{n, 2}}^{B_{2}} J_{t_{n, 1}}^{B_{1}}+\cdots+c_{M} J_{t_{n, M}}^{B_{M}} J_{t_{n, M-1}}^{B_{M-1}} \cdots J_{t_{n, 1}}^{B_{1}}$, and $J_{t_{n, j}}^{B_{j}}=\left(I+t_{n, j} B_{j}\right)^{-1}$, for $j=1,2, \ldots, M, 0<c_{k}<1$, for $k=0,1,2, \ldots, M$, and $\sum_{k=0}^{M} c_{k}=1$. Suppose $\left\{e_{n}\right\} \subset E,\left\{\alpha_{n}\right\},\left\{\beta_{n}\right\}$, and $\left\{\gamma_{n}\right\}$ are three sequences in $(0,1)$ and $\left\{r_{n, i}\right\},\left\{t_{n, j}\right\} \subset(0,+\infty)$ satisfy the following conditions:

(i) $\alpha_{n} \rightarrow 0, \beta_{n} \rightarrow 0$, as $n \rightarrow \infty$;

(ii) $\sum_{n=1}^{\infty} \alpha_{n} \beta_{n}=+\infty$;

(iii) $0<\liminf _{n \rightarrow+\infty} \gamma_{n} \leq \lim \sup _{n \rightarrow+\infty} \gamma_{n}<1$;

(iv) $\sum_{n=1}^{\infty}\left|r_{n+1, i}-r_{n, i}\right|<+\infty$ and $r_{n, i} \geq \varepsilon>0$, for $n \geq 1$ and $i=1,2, \ldots, N$;

(v) $\sum_{n=1}^{\infty}\left|t_{n+1, j}-t_{n, j}\right|<+\infty$ and $t_{n, j} \geq \varepsilon>0$, for $n \geq 1$ and $j=1,2, \ldots, M$;

(vi) $\frac{\left\|e_{n}\right\|}{\alpha_{n}} \rightarrow 0$, as $n \rightarrow+\infty$, and $\sum_{n=1}^{\infty}\left\|e_{n}\right\|<+\infty$.

Then $\left\{x_{n}\right\}$ converges strongly to a point $p_{0} \in D$.

Proof We shall split the proof into five steps:

Step 1. $\left\{x_{n}\right\},\left\{u_{n}\right\},\left\{S_{n} u_{n}\right\},\left\{v_{n}\right\},\left\{S_{n} v_{n}\right\}$ and $\left\{S_{n} x_{n}\right\}$ are all bounded.

Similar to the proof of step 1 in Theorem 3.1, we can get the result of step 1.

Then $\left\{J_{t_{n, 1}}^{B_{1}} S_{n} v_{n}\right\},\left\{J_{t_{n, 2}}^{B_{2}} J_{t_{n, 1}}^{B_{1}} S_{n} v_{n}\right\}, \ldots,\left\{J_{t_{n, M}}^{B_{M}} J_{t_{n, M-1}}^{B_{M-1}} \ldots J_{t_{n, 1}}^{B_{1}} S_{n} v_{n}\right\}$ are all bounded.

Step 2. $\lim _{n \rightarrow \infty}\left\|x_{n}-U_{n} S_{n} v_{n}\right\|=0$ and $\lim _{n \rightarrow \infty}\left\|x_{n+1}-x_{n}\right\|=0$. 
In fact,

$$
\begin{aligned}
& \left\|U_{n+1} S_{n+1} v_{n+1}-U_{n} S_{n} v_{n}\right\| \\
& \leq \\
& \quad c_{0}\left\|S_{n+1} v_{n+1}-S_{n} v_{n}\right\|+c_{1}\left\|J_{t_{n+1,1}}^{B_{1}} S_{n+1} v_{n+1}-J_{t_{n, 1}}^{B_{1}} S_{n} v_{n}\right\| \\
& \quad+c_{2}\left\|J_{t_{n+1,2}}^{B_{2}} J_{t_{n+1,1}}^{B_{1}} S_{n+1} v_{n+1}-J_{t_{n, 2}}^{B_{2}} J_{t_{n, 1}}^{B_{1}} S_{n} v_{n}\right\|+\cdots \\
& \quad+c_{M}\left\|J_{t_{n+1, M}}^{B_{M}} J_{t_{n+1, M-1}}^{B_{M-1}} \cdots J_{t_{n+1,1}}^{B_{1}} S_{n+1} v_{n+1}-J_{t_{n, M}}^{B_{M}} J_{t_{n, M-1}}^{B_{M-1}} \cdots J_{t_{n, 1}}^{B_{1}} S_{n} v_{n}\right\| .
\end{aligned}
$$

Similar to (3.13), we know that

$$
\left\|J_{t_{n+1,1}}^{B_{1}} S_{n+1} v_{n+1}-J_{t_{n, 1}}^{B_{1}} S_{n} v_{n}\right\| \leq\left\|S_{n+1} v_{n+1}-S_{n} v_{n}\right\|+2 M_{5} \frac{\left|t_{n+1,1}-t_{n, 1}\right|}{\varepsilon},
$$

where $M_{5}=\sup \left\{\left\|S_{n} v_{n}\right\|,\left\|J_{t_{n, 1}}^{B_{1}} S_{n} v_{n}\right\|,\left\|J_{t_{n, 2}}^{B_{2}} J_{t_{n, 1}}^{B_{1}} S_{n} v_{n}\right\|, \ldots,\left\|J_{t_{n, M}}^{B_{M}} J_{t_{n, M-1}}^{B_{M-1}} \ldots J_{t_{n, 1}}^{B_{1}} S_{n} v_{n}\right\|: n \geq 1\right\}$.

Repeating (3.22), we have

$$
\begin{aligned}
& \left\|J_{t_{n+1,2}}^{B_{2}} J_{t_{n+1,1}}^{B_{1}} S_{n+1} v_{n+1}-J_{t_{n, 2}}^{B_{2}} J_{t_{n, 1}}^{B_{1}} S_{n} v_{n}\right\| \\
& \quad \leq\left\|J_{t_{n+1,1}}^{B_{1}} S_{n+1} v_{n+1}-J_{t_{n, 1}}^{B_{1}} S_{n} v_{n}\right\|+\frac{2 M_{5}}{\varepsilon}\left|t_{n+1,2}-t_{n, 2}\right| .
\end{aligned}
$$

Then (3.22) and (3.23) imply that

$$
\begin{aligned}
& \left\|J_{t_{n+1,2}}^{B_{2}} J_{t_{n+1,1}}^{B_{1}} S_{n+1} v_{n+1}-J_{t_{n, 2}}^{B_{2}} J_{t_{n, 1}}^{B_{1}} S_{n} v_{n}\right\| \\
& \quad \leq\left\|S_{n+1} v_{n+1}-S_{n} v_{n}\right\|+\frac{2 M_{5}}{\varepsilon}\left(\left|t_{n+1,2}-t_{n, 2}\right|+\left|t_{n+1,1}-t_{n, 1}\right|\right) .
\end{aligned}
$$

By induction, we have

$$
\begin{aligned}
& \left\|J_{t_{n+1, M}}^{B_{M}} J_{t_{n+1, M-1}}^{B_{M-1}} \cdots J_{t_{n, 1}}^{B_{1}} S_{n+1} v_{n+1}-J_{t_{n, M}}^{B_{M}} J_{t_{n, M-1}}^{B_{M-1}} \cdots J_{t_{n, 1}}^{B_{1}} S_{n} v_{n}\right\| \\
& \quad \leq\left\|S_{n+1} v_{n+1}-S_{n} v_{n}\right\| \\
& \quad+\frac{2 M_{5}}{\varepsilon}\left(\left|t_{n+1, M}-t_{n, M}\right|+\cdots+\left|t_{n+1,2}-t_{n, 2}\right|+\left|t_{n+1,1}-t_{n, 1}\right|\right) .
\end{aligned}
$$

Going back to (3.21), we have

$$
\begin{aligned}
\| U_{n+1} & S_{n+1} v_{n+1}-U_{n} S_{n} v_{n} \| \\
\leq & \left\|S_{n+1} v_{n+1}-S_{n} v_{n}\right\| \\
& \quad+\frac{2 M_{5}}{\varepsilon}\left(\sum_{j=1}^{M} c_{j}\left|t_{n, 1}-t_{n+1,1}\right|+\sum_{j=2}^{M} c_{j}\left|t_{n, 2}-t_{n+1,2}\right|+\cdots+c_{M}\left|t_{n, M}-t_{n+1, M}\right|\right) .
\end{aligned}
$$

Therefore, similar to (3.17), we have

$$
\begin{aligned}
& \left\|U_{n+1} S_{n+1} v_{n+1}-U_{n} S_{n} v_{n}\right\| \\
& \quad \leq\left\|v_{n+1}-v_{n}\right\|+\frac{2 M_{2}}{\varepsilon} \sum_{i=1}^{N}\left|r_{n, i}-r_{n+1, i}\right|
\end{aligned}
$$




$$
\begin{aligned}
& +\frac{2 M_{5}}{\varepsilon}\left(\sum_{j=1}^{M} c_{j}\left|t_{n, 1}-t_{n+1,1}\right|+\sum_{j=2}^{M} c_{j}\left|t_{n, 2}-t_{n+1,2}\right|+\cdots+c_{M}\left|t_{n, M}-t_{n+1, M}\right|\right) \\
\leq & \left(1+\beta_{n}\right)\left\|x_{n+1}-x_{n}\right\|+\left(\beta_{n}+\alpha_{n} \beta_{n}\right)\left\|x_{n}\right\|+\left(\beta_{n+1}+\alpha_{n+1} \beta_{n}\right)\left\|x_{n+1}\right\| \\
& +\left|\beta_{n+1}-\beta_{n}\right|\left\|S_{n+1} u_{n+1}\right\|+\beta_{n}\left\|e_{n+1}-e_{n}\right\|+\beta_{n}\left\|\alpha_{n+1} e_{n+1}-\alpha_{n} e_{n}\right\| \\
& +\frac{4 M_{2}}{\varepsilon} \sum_{i=1}^{N}\left|r_{n, i}-r_{n+1, i}\right| \\
& +\frac{2 M_{5}}{\varepsilon}\left(\sum_{j=1}^{M} c_{j}\left|t_{n, 1}-t_{n+1,1}\right|+\sum_{j=2}^{M} c_{j}\left|t_{n, 2}-t_{n+1,2}\right|+\cdots+c_{M}\left|t_{n, M}-t_{n+1, M}\right|\right) .
\end{aligned}
$$

Thus limsup $\sup _{n \rightarrow+\infty}\left(\left\|U_{n+1} S_{n+1} v_{n+1}-U_{n} S_{n} v_{n}\right\|-\left\|x_{n+1}-x_{n}\right\|\right) \leq 0$. Using Lemma 2.5, we have from (3.27) $\lim _{n \rightarrow \infty}\left\|x_{n}-U_{n} S_{n} v_{n}\right\|=0$ and then $\lim _{n \rightarrow \infty}\left\|x_{n+1}-x_{n}\right\|=\lim _{n \rightarrow \infty}(1-$ $\left.\gamma_{n}\right)\left\|U_{n} S_{n} v_{n}-x_{n}\right\|=0$.

Similar to Theorem 3.1, we have

Step 3. $\lim _{n \rightarrow \infty}\left\|x_{n}-U_{n} S_{n} x_{n}\right\|=0$.

Step 4. lim $\sup _{n \rightarrow+\infty}\left\langle p_{0}, J\left(p_{0}-x_{n}\right)\right\rangle \leq 0$, where $p_{0}$ is an element in $D$.

From Lemma 3.8, we know that $U_{n} S_{n}: C \rightarrow C$ is nonexpansive and $F\left(U_{n} S_{n}\right)=D$. Then Lemma 3.1 and Lemma 3.3 imply that there exists $z_{t} \in C$ such that $z_{t}=U_{n} S_{n} Q_{C}\left[(1-t) z_{t}\right]$ for $t \in(0,1)$. Moreover, $z_{t} \rightarrow p_{0} \in D$, as $t \rightarrow 0$. Then copy step 4 in Theorem 3.1, the result follows.

Step 5. $x_{n} \rightarrow p_{0} \in D$, which is the same as that in step 4 .

Copy step 5 in Theorem 3.1, the result follows.

This completes the proof.

If in Theorem 3.3, $C=E$, then we have the following theorem.

Theorem 3.4 Let $E$ and $D$ be the same as those in Theorem 3.3. Suppose that the duality mapping $J: E \rightarrow E^{*}$ is weakly sequentially continuous. Let $A_{i}: E \rightarrow E(i=1,2, \ldots, N)$ and $B_{j}: E \rightarrow E(j=1,2, \ldots, M)$ be two finite families of m-accretive mappings. Let $\left\{e_{n}\right\} \subset E$, $\left\{\alpha_{n}\right\},\left\{\beta_{n}\right\},\left\{\gamma_{n}\right\} \subset(0,1)$ and $\left\{r_{n, i}\right\},\left\{t_{n, j}\right\} \subset(0,+\infty)$ satisfy the some conditions presented in Theorem 3.3.

Let $\left\{x_{n}\right\}$ be generated by the following scheme:

$$
\begin{aligned}
& x_{1} \in E, \\
& u_{n}=\left(1-\alpha_{n}\right)\left(x_{n}+e_{n}\right), \\
& v_{n}=\left(1-\beta_{n}\right) x_{n}+\beta_{n} S_{n} u_{n}, \\
& x_{n+1}=\gamma_{n} x_{n}+\left(1-\gamma_{n}\right) U_{n} S_{n} v_{n}, \quad n \geq 1 .
\end{aligned}
$$

Then $\left\{x_{n}\right\}$ converges strongly to a point $p_{0} \in D$, where $S_{n}$ and $U_{n}$ are the same as those in Theorem 3.3.

Next, we apply Theorems 3.1 and 3.3 to the cases of finite pseudo-contractive mappings.

Theorem 3.5 Let E be a real uniformly smooth and uniformly convex Banach space. Let $C$ be a nonempty, closed, and convex sunny nonexpansive retract of $E$, where $Q_{C}$ is the sunny 
nonexpansive retraction of $E$ onto $C$. Let $T_{i}^{(1)}, T_{j}^{(2)}: C \rightarrow$ E be pseudo-contractive mappings such that $\left(I-T_{i}^{(1)}\right)$ and $\left(I-T_{j}^{(2)}\right)$ are $m$-accretive, where $i=1,2, \ldots N, j=1,2, \ldots, M$. Suppose that the duality mapping $J: E \rightarrow E^{*}$ is weakly sequentially continuous and $D:=$ $\left(\bigcap_{i=1}^{N} F\left(T_{i}^{(1)}\right)\right) \cap\left(\bigcap_{j=1}^{M} F\left(T_{j}^{(2)}\right)\right) \neq \emptyset$. Let $\left\{x_{n}\right\}$ be generated by the iterative algorithm (A), where $S_{n}:=a_{0} I+a_{1} J_{r_{n, 1}}^{I-T_{1}^{(1)}}+a_{2} J_{r_{n, 2}}^{I-T_{2}^{(1)}}+\cdots+a_{N} J_{r_{n, N}}^{I-T_{N}^{(1)}}$, and $J_{r_{n, i}}^{I-T_{i}^{(1)}}=\left[I+r_{n, i}\left(I-T_{i}^{(1)}\right)\right]^{-1}$, for $i=1,2, \ldots, N, 0<a_{k}<1$, for $k=0,1,2, \ldots, N, \sum_{k=0}^{N} a_{k}=1 . W_{n}=b_{0} I+b_{1} J_{s_{n, 1}}^{I-T_{1}^{(2)}}+$ $b_{2} J_{s_{n, 2}}^{I-T_{2}^{(2)}}+\cdots+b_{M} J_{s_{n, M}}^{I-T_{M}^{(2)}}$, where $J_{s_{n, j}}^{I-T_{j}^{(2)}}=\left[I+s_{n, j}\left(I-T_{j}^{(2)}\right)\right]^{-1}$, for $j=1,2, \ldots, M, 0<b_{k}<1$, for $k=0,1,2, \ldots, M, \sum_{k=0}^{M} b_{k}=1$. Suppose $\left\{e_{n}\right\} \subset E,\left\{\alpha_{n}\right\},\left\{\beta_{n}\right\}$, and $\left\{\gamma_{n}\right\}$ are three sequences in $(0,1)$ and $\left\{r_{n, i}\right\},\left\{s_{n, j}\right\} \subset(0,+\infty)$ satisfying the following conditions:

(i) $\alpha_{n} \rightarrow 0, \beta_{n} \rightarrow 0$, as $n \rightarrow \infty$;

(ii) $\sum_{n=1}^{\infty} \alpha_{n} \beta_{n}=+\infty$;

(iii) $0<\liminf _{n \rightarrow+\infty} \gamma_{n} \leq \lim \sup _{n \rightarrow+\infty} \gamma_{n}<1$;

(iv) $\sum_{n=1}^{\infty}\left|r_{n+1, i}-r_{n, i}\right|<+\infty$ and $r_{n, i} \geq \varepsilon>0$, for $n \geq 1$ and $i=1,2, \ldots, N$;

(v) $\sum_{n=1}^{\infty}\left|s_{n+1, j}-s_{n, j}\right|<+\infty$ and $s_{n, j} \geq \varepsilon>0$, for $n \geq 1$ and $j=1,2, \ldots, M$;

(vi) $\frac{\left\|e_{n}\right\|}{\alpha_{n}} \rightarrow 0$, as $n \rightarrow+\infty$, and $\sum_{n=1}^{\infty}\left\|e_{n}\right\|<+\infty$.

Then $\left\{x_{n}\right\}$ converges strongly to a point $p_{0} \in D$.

Proof Let $A_{i}=\left(I-T_{i}^{(1)}\right)$ and $B_{j}=\left(I-T_{j}^{(2)}\right)$, for $i=1,2, \ldots, N$ and $j=1,2, \ldots, M$. Then the result follows from Theorem 3.1.

Similarly, from Theorem 3.3, we have the following result.

Theorem 3.6 Let E, C, $Q_{C}$ and $D$ be the same as those in Theorem 3.5. Let $T_{i}^{(1)}, T_{j}^{(2)}$ : $C \rightarrow E$ be pseudo-contractive mappings such that $\left(I-T_{i}^{(1)}\right)$ and $\left(I-T_{j}^{(2)}\right)$ are $m$-accretive mappings, where $i=1,2, \ldots N, j=1,2, \ldots, M$. Suppose that the duality mapping $J: E \rightarrow E^{*}$ is weakly sequentially continuous and $D \neq \emptyset$. Let $\left\{x_{n}\right\}$ be generated by the iterative algorithm (B), where $S_{n}$ is the same as that in Theorem 3.5 and $U_{n}=c_{0} I+c_{1} J_{t_{n, 1}}^{I-T_{1}^{(2)}}+$ $c_{2} J_{t_{n, 2}}^{I-T_{2}^{(2)}} J_{t_{n, 1}}^{I-T_{1}^{(2)}}+\cdots+c_{M} J_{t_{n, M}}^{I-T_{M}^{(2)}} J_{t_{n, M-1}}^{I-T_{M-1}^{(2)}} \cdots J_{t_{n, 1}}^{I-T_{1}^{(2)}}$, where $J_{t_{n, j}}^{I-T_{j}^{(2)}}=\left[I+t_{n, j}\left(I-T_{j}^{(2)}\right)\right]^{-1}$, for $j=$ $1,2, \ldots, M, 0<c_{k}<1$, for $k=0,1,2, \ldots, M, \sum_{k=0}^{M} c_{k}=1$. Suppose $\left\{e_{n}\right\} \subset E,\left\{\alpha_{n}\right\},\left\{\beta_{n}\right\}$, and $\left\{\gamma_{n}\right\}$ are three sequences in $(0,1)$ and $\left\{r_{n, i}\right\},\left\{t_{n, j}\right\} \subset(0,+\infty)$ satisfying the following conditions:

(i) $\alpha_{n} \rightarrow 0, \beta_{n} \rightarrow 0$, as $n \rightarrow \infty$;

(ii) $\sum_{n=1}^{\infty} \alpha_{n} \beta_{n}=+\infty$;

(iii) $0<\liminf _{n \rightarrow+\infty} \gamma_{n} \leq \lim \sup _{n \rightarrow+\infty} \gamma_{n}<1$;

(iv) $\sum_{n=1}^{\infty}\left|r_{n+1, i}-r_{n, i}\right|<+\infty$ and $r_{n, i} \geq \varepsilon>0$, for $n \geq 1$ and $i=1,2, \ldots, N$;

(v) $\sum_{n=1}^{\infty}\left|t_{n+1, j}-t_{n, j}\right|<+\infty$ and $s_{n, j} \geq \varepsilon>0$, for $n \geq 1$ and $j=1,2, \ldots, M$;

(vi) $\frac{\left\|e_{n}\right\|}{\alpha_{n}} \rightarrow 0$, as $n \rightarrow+\infty$, and $\sum_{n=1}^{\infty}\left\|e_{n}\right\|<+\infty$.

Then $\left\{x_{n}\right\}$ converges strongly to a point $p_{0} \in D$. 


\section{Acknowledgements}

This paper is supported by the National Natural Science Foundation of China (No. 11071053), Natural Science Foundation of Hebei Province (No. A2014207010), Key Project of Science and Research of Hebei Educational Department (ZH2012080) and Key Project of Science and Research of Hebei University of Economics and Business (2013KYZO1).

Received: 21 February 2014 Accepted: 31 July 2014 Published: 18 August 2014

\section{References}

1. Takahashi, W: Proximal point algorithms and four resolvents of nonlinear operators of monotone type in Banach spaces. Taiwan. J. Math. 12(8), 1883-1910 (2008)

2. Shehu, Y, Ezeora, JN: Path convergence and approximation of common zeroes of a finite family of $m$-accretive mappings in Banach spaces. Abstr. Appl. Anal. 2010, Article ID 285376 (2010)

3. Takahashi, W: Nonlinear Functional Analysis. Fixed Point Theory and Its Application. Yokohama Publishers, Yokohama (2000)

4. Rockafellar, RT: Monotone operators and the proximal point algorithm. SIAM J. Control Optim. 14(5), 877-898 (1976)

5. Chen, RD, Liu, YJ, Shen, XL: Iterative approximation of a zero of accretive operator in Banach space. Nonlinear Anal. 71, e346-e350 (2009)

6. Qin, XL, Su, YF: Approximation of a zero point of accretive operator in Banach spaces. J. Math. Anal. Appl. 329 415-424 (2007)

7. Mainge, PE: Viscosity methods for zeroes of accretive operators. J. Approx. Theory 140, 127-140 (2006)

8. Qin, XL, Cho, SY, Wang, L: Iterative algorithms with errors for zero points of $m$-accretive operators. Fixed Point Theory Appl. 2013, Article ID 148 (2013)

9. Ceng, LC, Wu, SY, Yao, JC: New accuracy criteria for modified approximate proximal point algorithms in Hilbert spaces. Taiwan. J. Math. 12(7), 1691-1705 (2008)

10. Xu, HK: Strong convergence of an iterative method for nonexpansive and accretive operators. J. Math. Anal. Appl. 314, 631-643 (2006)

11. Cho, JC, Kang, SM, Zhou, HY: Approximate proximal point algorithms for finding zeroes of maximal monotone operator in Hilbert spaces. J. Inequal. Appl. 2008, Article ID 598191 (2008)

12. Ceng, LC, Khan, AR, Ansari, QH, Yao, JC: Strong convergence of composite iterative schemes for zeros of $m$-accretive operators in Banach spaces. Nonlinear Anal. 70, 1830-1840 (2009)

13. Halpern, B: Fixed points of nonexpansive maps. Bull. Am. Math. Soc. 73, 957-961 (1967)

14. Zegeye, $\mathrm{H}$, Shahzad, $\mathrm{N}$ : Strong convergence theorems for a common zero of a finite family of $m$-accretive mappings. Nonlinear Anal. 66, 1161-1169 (2007)

15. $\mathrm{Hu}, \mathrm{LG}, \mathrm{Liu}, \mathrm{LW}: \mathrm{A}$ new iterative algorithm for common solutions of a finite family of accretive operators. Nonlinear Anal. 70, 2344-2351 (2009)

16. Yao, Y, Liou, YC, Marino, G: Strong convergence of two iterative algorithms for nonexpansive mappings in Hilbert spaces. Fixed Point Theory Appl. 2009, Article ID 279058 (2009)

17. Reich, S: Approximating fixed points of nonexpansive mappings. Panam. Math. J. 4(2), 23-28 (1994)

18. Wei, L, Tan, RL: Iterative scheme with errors for common zeros of finite accretive mappings and nonlinear elliptic systems. Abstr. Appl. Anal. 2014, Article ID 646843 (2014)

19. Browder, FE: Nonexpansive nonlinear operators in a Banach space. Proc. Natl. Acad. Sci. USA 54, 1041-1044 (1965)

20. Xu, HK: Iterative algorithms for nonlinear operators. J. Lond. Math. Soc. 66, 240-256 (2002)

21. Suziki, T: Strong convergence theorems for infinite families of nonexpansive mappings in general Banach spaces. Fixed Point Theory Appl. 2005, Article ID 685918 (2005)

22. Barbu, V: Nonlinear Semigroups and Differential Equations in Banach Space. Noordhoff, Leyden (1976)

doi:10.1186/1687-1812-2014-176

Cite this article as: Wei and Tan: Some new iterative algorithms with errors for common solutions of two finite families of accretive mappings in a Banach space. Fixed Point Theory and Applications 2014 2014:176.

\section{Submit your manuscript to a SpringerOpen ${ }^{\circ}$ journal and benefit from:}

- Convenient online submission

Rigorous peer review

- Immediate publication on acceptance

- Open access: articles freely available online

- High visibility within the field

- Retaining the copyright to your article 